Internat. J. Math. \& Math. Sci.

Vol. 23, No. 9 (2000) 585-595

S0161171200002969

(C) Hindawi Publishing Corp.

\title{
ON INVERTOR ELEMENTS AND FINITELY GENERATED SUBGROUPS OF GROUPS ACTING ON TREES WITH INVERSIONS
}

\author{
R. M. S. MAHMOOD and M. I. KHANFAR
}

(Received 9 February 1999)

\begin{abstract}
An element of a group acting on a graph is called invertor if it transfers an edge of the graph to its inverse. In this paper, we show that if $G$ is a group acting on a tree $X$ with inversions such that $G$ does not fix any element of $X$, then an element $g$ of $G$ is invertor if and only if $g$ is not in any vertex stabilizer of $G$ and $g^{2}$ is in an edge stabilizer of $G$. Moreover, if $H$ is a finitely generated subgroup of $G$, then $H$ contains an invertor element or some conjugate of $H$ contains a cyclically reduced element of length at least one on which $H$ is not in any vertex stabilizer of $G$, or $H$ is in a vertex stabilizer of $G$.
\end{abstract}

Keywords and phrases. Groups acting on trees, invertor elements, finitely generated subgroups.

2000 Mathematics Subject Classification. Primary 20F65, 20 E06.

1. Introduction. Lyndon and Schupp [5, Lemma 6.8, page 212] proved that if $G$ is a nontrivial free product with amalgamation, then for any finitely generated subgroup $H$ of $G, H$ is contained in a conjugate of a factor of $G$ or some conjugate of $H$ contains a cyclically reduced element of length at least two. Similarly, if $G$ is an $H N N$ group then, for any finitely generated subgroup $H$ of $G, H$ is contained in a conjugate of the base or some conjugate of $H$ contains a cyclically reduced element of length at least two. These results can be easily generalized to the result that finitely generated subgroups of groups acting on nontrivial trees without inversions are contained in a vertex stabilizers or some of their conjugates contain cyclically reduced elements of length at least one. In this paper, we show that the situation of groups acting on trees with inversions is different in the sense that if $G$ is a group acting on a tree $X$ with inversions such that $G$ does not fix any element of $X$ and if $H$ is a finitely generated subgroup of $G$, then $H$ is contained in a vertex stabilizer of $G$ or a conjugate of $H$ contains a cyclically reduced element of length at least one or $H$ contains an invertor element on which $H$ is not in any vertex stabilizer of $G$. This paper is arranged as follows. In Section 2, we give preliminary definitions and results needed for the rest of sections. In Section 3, we discuss some properties of invertor elements. In Section 4, we discuss finitely generated subgroups of groups acting on trees. In Section 5, we apply the results of previous sections to new groups called quasi HNN groups that are generalization of $H N N$ groups.

2. Preliminary definitions and results. By a graph $X$ we understand a pair of disjoint sets $V(X)$ called the set of vertices and $E(X)$ called the set of edges with $V(X)$ 
nonempty, equipped with two mappings $E(X) \rightarrow V(X) \times V(X), y \rightarrow(o(y), t(y))$ and, $E(X) \rightarrow E(X), y \rightarrow \bar{y}$ satisfying the conditions $\overline{\bar{y}}=y$ and $o(\bar{y})=t(y)$ for all $y \in E(X)$. The case $\bar{y}=y$ is possible for some $y \in E(X)$. For $y \in E(X), o(y)$ and $t(y)$ are called the ends of $y$ and $\bar{y}$ is called the inverse of $y$. There are obvious definitions of trees, morphism of graphs and Aut $(X)$, the set of all automorphisms of the graph $X$ which is a group under the composition of morphisms. For more details we refer the readers to Mahmood [6, 7] or Serre [10]. We say that a group $G$ acts on a graph $X$ if there is a group homomorphism $\phi: G \rightarrow \operatorname{Aut}(X)$. If $x \in X$ (vertex or edge) and $g \in G$, we write $g(x)$ for $(\phi(g))(x)$. Thus, if $g \in G$ and $y \in E(X)$, then $g(o(y))=o(g(y)), g(t(y))=t(g(y))$, and $g(\bar{y})=\overline{g(y)}$. The case $g(y)=\bar{y}$ for some $g \in G$ and $y \in E(X)$ may occur. That is, $G$ acts with inversions on $X$. We have the following notations related to the action of the group $G$ on the graph $X$.

(1) If $x \in X$ (vertex or edge), define $G(x)$ to be the set $G(x)=\{g(x): g \in G\}$. This set is called the orbit of $X$ containing $x$.

(2) If $x, y \in X$ define $G(x, y)$ to be the set $G(x, y)=\{g \in G: g(x)=y\}$, and $G(x, x)=G_{x}$, the stabilizer of $x$. Thus, $G(x, y) \neq \varphi$ if and only if $x$ and $y$ are in the same orbit. If $y \in E(X)$ and $u \in\{o(y), t(y)\}$, then it is clear that $G_{\bar{y}}=G_{y}$ and $G_{y} \leq G_{u}$.

(3) The set of elements of $X$ fixed by $G$ is denoted by $X^{G}$. That is, $X^{G}=\{x \in X$ : $\left.G_{x}=G\right\}$.

(4) If $X$ is connected, then a subtree $T$ of $X$ is called a tree of representatives for the action of $G$ on $X$ if $T$ contains exactly one vertex from each vertex orbit, and the subgraph $Y$ of $X$ containing a tree of representatives $T$, say, is called a fundamental domain for the action of $G$ on $X$ if each edge of $Y$ has at least one end in $T$, and $Y$ contains exactly one edge $y$, say, from each edge orbit such that $G(y, \bar{y})=\varphi$, and exactly one pair $x, \bar{x}$ from each edge orbit such that $G(x, \bar{x}) \neq \varphi$. For the existence of $T$ and $Y$ (see Khanfar and Mahmood [4]).

Henceforth, $G$ will be a group acting on a tree $X, T$ a tree of representatives for the action of $G$ on $X$, and $Y$ a fundamental domain for the action of $G$ on $X$ such that $X^{G}=\varphi$ and $T \subseteq Y$.

Definition 2.1. For any vertex $v$ of $X$ define $v^{*}$ to be the unique vertex of $T$ such that $G\left(v, v^{*}\right) \neq \varphi$. That is, $v$ and $v^{*}$ are in the same vertex orbit. It is clear that if $v$ is in $T$, then $v^{*}=v$ and in general $v^{* *}=v^{*}$. Moreover, if $u$ and $v$ are two vertices of $X$ such that $G(u, v) \neq \varphi$, then $u^{*}=v^{*}$.

DEFINITION 2.2. For each edge $y$ of $Y$ define the following.

(1) Define $[y]$ to be an element of $G\left(t(y), t(y)^{*}\right)$. That is, $[y]\left((t(y))^{*}\right)=t(y)$ to be chosen as follows:

(a) if $o(y) \in V(T)$, then $[y]=1$ in case $y \in E(T)$, and $[y](y)=\bar{y}$ if $G(y, \bar{y}) \neq \varphi$,

(b) if $o(y) \notin V(T)$, then $[y]=[\bar{y}]^{-1}$ if $G(y, \bar{y})=\varphi$, otherwise $[y]=[\bar{y}]$ if $G(y, \bar{y}) \neq \varphi$.

(2) Define $-y$ to be the edge $-y=[y]^{-1}(y)$ if $o(y) \in V(T)$, otherwise $-y=y$, and define $+y$ to be the edge $+y=[y](-y)$. It is clear that $t(-y)=(t(y))^{*}, o(+y)=$ $(o(y))^{*},(\overline{-y})=+(\bar{y}),(\overline{+y})=-(\bar{y}), G_{-y} \leq G_{(t(y)) *}$, and $G_{+y} \leq G_{(o(y)) *}$. Moreover, if $G(y, \bar{y}) \neq \varphi$, or $y \in E(T)$, then $G_{-y}=G_{+y}=G_{y}$. 
(3) Define $\phi_{y}$ to be the map $\phi_{y}: G_{-y} \rightarrow G_{+y}$ given by $\phi_{y}(g)=[y] g[y]^{-1}$. It is clear that $\phi_{y}$ is an isomorphism.

(4) Define $\delta_{y}$ to be the element $\delta_{y}=[y][\bar{y}]$. It is clear that $\delta_{y}=1$ if $G(y, \bar{y})=\varphi$. Otherwise $\delta_{y}=[y]^{2}$. Consequently $\delta_{y} \in G_{y}, \delta_{\bar{y}}=\delta_{y}$ and $\phi_{y}\left(\delta_{y}\right)=\delta_{y}$.

Proposition 2.3. $G$ is generated by the generators of $G_{v}$ and by the elements $[y]$, where $v$ runs over $V(T)$ and $y$ runs over $E(Y)$.

Proof. See Mahmood [6].

DEFINITION 2.4. By a word $w$ of $G$ we mean an expression of the form

$$
\begin{gathered}
w=g_{0} \cdot y_{1} \cdot g_{1} \cdot y_{2} \cdot g_{2} \cdots y_{n} \cdot g_{n}, \quad n \geq 0, \\
y_{i} \in E(Y), \quad \text { for } i=1,2, \ldots, n
\end{gathered}
$$

such that

(1) $g_{0} \in G_{\left(o\left(y_{1}\right)\right) * \text {; }}$

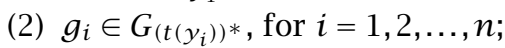

(3) $\left(t\left(y_{i}\right)\right)^{*}=\left(o\left(y_{i+1}\right)\right)^{*}$, for $i=1,2, \ldots, n-1$.

We define $o(w)=\left(o\left(y_{1}\right)\right)^{*}$ and $t(w)=\left(t\left(y_{n}\right)\right)^{*}$.

If $o(w)=t(w)$, then $w$ is called a closed word of $G$ of type $v, v=o(w)$.

We have the following definitions related to the word $w$ defined above:

(i) $n$ is called the length of $w$ and is denoted by $|w|=n$.

(ii) $w$ is called a trivial word of $G$ if $|w|=0$, or $w=g_{0}$.

(iii) The value of $w$ denoted [ $w$ ] is defined to be the element

$$
[w]=g_{0}\left[y_{1}\right] g_{1}\left[y_{2}\right] g_{2} \cdots\left[y_{n}\right] g_{n} \quad \text { of } G .
$$

(iv) The inverse of $w$ denoted $w^{-1}$ is defined to be the word

$$
w^{-1}=g_{n}^{-1} \cdot \bar{y}_{n} \cdot \delta_{y_{n}}^{-1} g_{n-1}^{-1} \cdots g_{2}^{-1} \cdot \bar{y}_{2} \cdot \delta_{y_{2}}^{-1} g_{1}^{-1} \cdot \bar{y}_{1} \cdot \delta_{y_{1}}^{-1} g_{0}^{-1} \quad \text { of } G .
$$

It is clear that $\left[w^{-1}\right]=[w]^{-1}$ but $\left(w^{-1}\right)^{-1} \neq w$ if $w$ contains an edge $y$ (say) such that $G(y, \bar{y}) \neq \varphi$. Otherwise $\left(w^{-1}\right)^{-1}=w$.

(v) If $w^{\prime}=h_{0} \cdot x_{1} \cdot h_{1} \cdot x_{2} \cdot h_{2} \cdots x_{m} \cdot h_{m}$ is a word of $G$ such that $t(w)=o\left(w^{\prime}\right)$, then $w \cdot w^{\prime}$ is defined to be the word

$$
w \cdot w^{\prime}=g_{0} \cdot y_{1} \cdot g_{1} \cdot y_{2} \cdot g_{2} \cdots y_{n} \cdot g_{n} h_{0} \cdot x_{1} \cdot h_{1} \cdot x_{2} \cdot h_{2} \cdots x_{m} \cdot h_{m} \quad \text { of } G .
$$

It is clear that $\left[w \cdot w^{\prime}\right]=[w]\left[w^{\prime}\right], o\left(w \cdot w^{\prime}\right)=o(w)$, and $t\left(w \cdot w^{\prime}\right)=t\left(w^{\prime}\right)$.

(vi) $w$ is called a reduced word of $G$ if $w$ contains no subword of the forms

(1) $y_{i} \cdot g_{i} \cdot \bar{y}_{i}$ if $g_{i} \in G_{-y_{i}}$ for $i=1, \ldots, n$,

(2) $y_{i} \cdot g_{i} \cdot y_{i}$ if $g_{i} \in G_{y_{i}}$ and $G\left(y_{i}, \bar{y}_{i}\right) \neq \varphi$ for $i=1, \ldots, n$.

We take trivial words to be reduced.

DEFINITION 2.5. By the edge reduction on the word

$$
w=g_{0} \cdot y_{1} \cdot g_{1} \cdot y_{2} \cdot g_{2} \ldots \cdot y_{n} \cdot g_{n}
$$

we mean the performance of the following operations on $w$ : 
(1) replace a subword of the form $y_{i} \cdot g_{i} \cdot \bar{y}_{i}$, if $g_{i} \in G_{-y_{i}}$, by $\phi_{y_{i}}\left(g_{i} \delta_{y_{i}}\right)$,

(2) replace a subword of the form $y_{i} \cdot g_{i} \cdot y_{i}$, if $g_{i} \in G_{y_{i}}$ and $G\left(y_{i}, \bar{y}_{i}\right) \neq \varphi$, by $\phi_{y_{i}}\left(g_{i} \delta_{y_{i}}\right)$.

We define $w^{0}$ to be the identity element 1 of $G$.

THEOREM 2.6. Every element of $G$ is the value of a closed and reduced word of $G$. Moreover, if $w$ a closed word of $G$ of value 1 , the identity element of $G$, then $w$ is not reduced.

Proof. See Mahmood [7].

Now we generalize Theorem 2.6 as follows.

LEMMA 2.7. For every element $g$ of $G$ and any two vertices $u$ and $v$ of $T$ there exists a reduced word $w$ of $G$ such that $o(w)=u, t(w)=v$, and $[w]=g$. Moreover, if $w$ is reduced, then for any word $w^{\prime}$ of $G$ such that $o(w)=o\left(w^{\prime}\right), t(w)=t\left(w^{\prime}\right)$, and $[w]=\left[w^{\prime}\right]=g$, we have $|w|=\left|w^{\prime}\right|$ if and only if $w^{\prime}$ is a reduced.

Proof. Since $G$ is generated by the generators of $G_{z}$ and by the elements $[y]$, where $z$ runs over the vertices of $T$ and $y$ over the edges of $Y$, therefore $g$ can be written as the product $g=g_{0}\left[y_{1}\right] g_{1}\left[y_{2}\right] g_{2} \cdots\left[y_{n}\right] g_{n}$, where $g_{i} \in G_{u_{i}}$ for some vertices $u_{0}, u_{1}, \ldots, u_{n}$ of $T$, and edges $y_{1}, y_{2}, \ldots, y_{n}$ of $Y$. By taking the reduced paths in $T$ between $u$ and $u_{i}$, between $u_{i}$ and $\left(o\left(y_{i}\right)\right)^{*}$, and between $\left(t\left(y_{i}\right)\right)^{*}$ and $v$, and the identity element 1 of $G$ we may choose this product so that $w=g_{0} \cdot y_{1} \cdot g_{1} \cdot y_{2} \cdot g_{2} \cdots y_{n} \cdot g_{n}$ is a word of $G$ such that $o(w)=u, t(w)=v$, and $[w]=g$. If $w$ is not reduced then for some $i, 1 \leq i \leq n-1$ we have $y_{i+1}=\bar{y}_{i}$ and $g_{i} \in G_{-y_{i}}$ or $y_{i+1}=y_{i}, g_{i} \in G_{y_{i}}$, and $G\left(y_{i}, \bar{y}_{i}\right) \neq \varphi$. If $y_{i+1}=\bar{y}_{i}$ and $g_{i} \in G_{-y_{i}}$, then we replace $y_{i} \cdot g_{i} \cdot \bar{y}_{i}$ by the element $\phi_{y_{i}}\left(g_{i} \delta_{y_{i}}\right)$ in $w$. We get a new word $g_{0} \cdot y_{1} \cdot g_{1} \cdot y_{2} \cdot g_{2} \cdots y_{i-1} \cdot g_{i-1} \phi_{y_{i}}\left(g_{i} \delta_{y_{i}}\right) \cdot y_{i+1}$. $\cdots y_{n} \cdot g_{n}$.

If $y_{i+1}=y_{i}, g_{i} \in G_{y i}$, and $G\left(y_{i}, \bar{y}_{i}\right) \neq \varphi$, then we replace $y_{i} \cdot g_{i} \cdot y_{i+1}$ by the element $\phi_{y_{i}}\left(g_{i} \delta_{y_{i}}\right)$ in $w$. We get a new word as above.

Continuing the above processes on $w$ yields a reduced word of $G$ satisfying the required properties. In other words, the performance of the edge reductions on $w$ yields a reduced word of $G$ of value [ $w$ ].

Now let $w=g_{0} \cdot y_{1} \cdot g_{1} \cdot y_{2} \cdot g_{2} \cdots y_{n} \cdot g_{n}, n \geq 0$ and

$$
w^{\prime}=h_{0} \cdot x_{1} \cdot h_{1} \cdot x_{2} \cdot h_{2} \cdots x_{m} \cdot h_{m}
$$

be two words of $G$ such that $w$ is reduced $o(w)=o\left(w^{\prime}\right), t(w)=t\left(w^{\prime}\right)$ and $[w]=$ $\left[w^{\prime}\right]=g$. Assume that $w^{\prime}$ is reduced. We need to show that $n=m$. Since $\left[w^{\prime}\right][w]^{-1}=$ 1 , the identity of $G$, therefore by Theorem 2.6 , the word

$$
w_{0}=h_{0} \cdot x_{1} \cdot h_{1} \cdot x_{2} \cdot h_{2} \cdots x_{m} \cdot h_{m} g_{n}^{-1} \cdot \bar{y}_{n} \cdot \delta_{y_{n}}^{-1} g_{n-1}^{-1} \cdots \cdot g_{2}^{-1} \cdot \bar{y}_{2} \cdot \delta_{y_{2}}^{-1} g_{1}^{-1} \cdot \bar{y}_{1} \cdot \delta_{y_{1}}^{-1} g_{0}^{-1}
$$

is not reduced. Since $w$ and $w^{\prime}$ are reduced, therefore $y_{n}=x_{m}$ and $h_{m} g_{n}^{-1} \in G_{-y_{n}}$, or $y_{n}=\bar{x}_{m}$ and $h_{m} \mathcal{g}_{n}^{-1} \in G_{y_{n}}$ if $G\left(y_{n}, \bar{y}_{n}\right) \neq \varphi$. In both cases, we substitute 
$x_{m} \cdot h_{m} \mathcal{g}_{n}^{-1} \cdot \bar{y}_{n}$ or $\left(x_{m} \cdot h_{m} \mathcal{g}_{n}^{-1} \cdot y_{n}\right)$ by $\phi_{y_{n}}\left(h_{m} \mathcal{g}_{n}^{-1} \delta_{y_{n}}\right)$ in $w_{0}$. We get a new word $w_{1}=h_{0} \cdot x_{1} \cdot h_{1} \cdot x_{2} \cdot h_{2} \cdots x_{m-1} \cdot h_{m-1} A \delta_{y_{n}}^{-1} g_{n-1}^{-1} \cdot \bar{y}_{n-1} \cdots g_{2}^{-1} \cdot \bar{y}_{2} \cdot \delta_{y_{2}}^{-1} g_{1}^{-1} \cdot \bar{y}_{1} \cdot \delta_{y_{1}}^{-1} g_{0}^{-1}$,

where $A=\phi_{y_{n}}\left(h_{m} g_{n}^{-1} \delta_{y_{n}}\right)$. Then $w_{1}$ is not reduced. Similarly, we have $x_{m-1}=\bar{y}_{n-1}$, and $h_{m-1} \phi_{y_{n}}\left(h_{m} g_{n}^{-1} \delta_{y_{n}}\right) \delta_{y_{n}}^{-1} g_{n-1}^{-1} \in G_{y_{n-1}}$ or $x_{m-1}=y_{n-1}$ if $G\left(y_{n-1}, \bar{y}_{n-1}\right) \neq \varphi$.

Now continuing above processes yields $x_{1}=y_{1}$ or $x_{1}=\bar{y}_{1}$ if $G\left(y_{1}, \bar{y}_{1}\right) \neq \varphi$. This implies that $|w|=\left|w^{\prime}\right|$.

Conversely, assume that $w$ is reduced and $|w|=\left|w^{\prime}\right|$. We need to show that $w^{\prime}$ is reduced. For, if $w^{\prime}$ is not reduced then by applying edge reductions on $w^{\prime}$ yields a reduced word $w^{\prime \prime}$ of $G$ such that $o\left(w^{\prime}\right)=o\left(w^{\prime \prime}\right), t\left(w^{\prime}\right)=t\left(w^{\prime \prime}\right),\left[w^{\prime}\right]=\left[w^{\prime \prime}\right]$ and $\left|w^{\prime \prime}\right|<\left|w^{\prime}\right|$. Contradiction. Hence $w^{\prime}$ is reduced. This completes the proof.

DEFINITION 2.8. For each element $g$ of $G$ and each vertex $v$ of $V(T)$ define $|g|_{v}$ to be the length of a reduced word of $G$ of type $v$ and value $g$. In view of Theorem 2.6 and Lemma 2.7, this concept is clear. $|g|_{v}$ is called the length of $g$ with respect to $v$.

Proposition 2.9. Let $g$ be an element of $G$ and $v$ a vertex of $V(T)$ such that $|g|_{v}$ is even. Then for any vertex $u$ of $V(T),|g|_{u}$ is even.

Proof. Let $w_{1}$ and $w_{2}$ be two reduced and closed words of $G$ of types $v$ and $u$ respectively such that $w_{1}$ and $w_{2}$ are of value $g$ and $\left|w_{1}\right|$ is even. We need to show that $\left|w_{2}\right|$ is even. Let $w_{0}$ be the word

$$
w_{0}=1 \cdot y_{1} \cdot 1 \cdot y_{2} \cdot 1 \cdots y_{n} \cdot 1 \cdot w_{1} \cdot 1 \cdot \bar{y}_{n} \cdot 1 \cdots \cdot 1 \cdot \bar{y}_{2} \cdot 1 \cdot \bar{y}_{1} \cdot 1,
$$

where $y_{1}, y_{2}, \ldots, y_{n}$ is the reduced path in $T$ joining $u$ and $v$. It is clear that $w_{0}$ is a word of $G$ of type $u$ and of value $\left[w_{2}\right]$, and $w_{0}$ is of even length. By applying edge reductions on $w_{0}$ yields a reduced word of $G$ of type $u$, value $\left[w_{2}\right]$ and of even length. Then Lemma 2.7 implies that $\left|w_{2}\right|$ is even. This completes the proof.

Proposition 2.10. If $g$ is an element of $G_{v}$ for some vertex $v$ of $V(X)$, then $|g|_{u}$ is even for any vertex $u$ of $V(T)$.

Proof. $g=a b a^{-1}$ where $a \in G$, and $b \in G_{v^{*}}$. Let $u$ be any vertex of $V(T)$ and $w$ a reduced word of $G$ such that $o(w)=u, t(u)=v^{*}$, and $[w]=a$. Then the word $w_{0}=w \cdot b \cdot w^{-1}$ is closed of type $u$, value $g$, and is of even length. Now applying edge reductions on $w_{0}$ yields a reduced word of $G$ of type $u$, value $g$, and of even length. This implies that $|g|_{u}$ is even. This completes the proof.

DEFINITION 2.11. Let $w$ be a closed and reduced word of $G$. We say that $w$ is a cyclically reduced word of $G$ if $w^{2}$ is reduced.

It is clear that every word of length zero is a cyclically reduced.

The proof of the following proposition is clear.

Proposition 2.12. Let $w=g_{0} \cdot y_{1} \cdot g_{1} \cdot y_{2} \cdot g_{2} \cdots y_{n} \cdot g_{n}, n>0$ be a closed and reduced word of $G$. Then the following are equivalent:

(1) $w^{m}$ is cyclically reduced for every integer $m$,

(2) every cyclic permutation of $w$ is reduced, 
(3) $g_{n-1} \cdot y_{n} \cdot g_{n} g_{0} \cdot y_{1} \cdot g_{1}$ is a reduced word of $G$,

(4) $\left|w^{m}\right|=|m||w|$ for every integer $m$,

(5) $\left|w^{m}\right|<\left|w^{m+1}\right|$ for every integer $m, m \geq 0$.

Proposition 2.13. Let $w_{1}$ and $w_{2}$ be two closed and reduced words of $G$ such that $w_{1}$ and $w_{2}$ are of the same type and of the same value, and $w_{1}$ be cyclically reduced. Then $w_{2}$ is cyclically reduced. Moreover, if $\left|w_{1}\right| \geq 1$, then $\left[w_{1}\right]$ is not in any vertex stabilizer of $G$.

Proof. By Lemma 2.7, $\left|w_{1}\right|=\left|w_{2}\right|$. If $\left|w_{1}\right|=0$, then by definition $w_{2}$ is cyclically reduced. Let $w_{1}=g_{0} \cdot y_{1} \cdot g_{1} \cdot y_{2} \cdot g_{2} \ldots y_{n} \cdot g_{n}, n>0$ and

$$
w_{2}=h_{0} \cdot x_{1} \cdot h_{1} \cdot x_{2} \cdot h_{2} \cdots \cdot x_{n} \cdot h_{n} .
$$

We need to show that $1 \cdot x_{n} \cdot h_{n} h_{0} \cdot x_{1} \cdot 1$ is reduced. Since $w_{1}$ is cyclically reduced, therefore $g_{n} g_{0} \notin G_{-y_{n}}$. By Lemma $2.7, h_{n} h_{0} \notin G_{-y_{n}}$. So $1 \cdot x_{n} \cdot h_{n} h_{0} \cdot x_{1} \cdot 1$ is reduced. Therefore $w_{2}$ is cyclically reduced. Now assume that $\left|w_{1}\right| \geq 1$. If $\left[w_{1}\right] \in G_{v}$ for $v \in V(X)$, then $\left[w_{1}\right]=a b a^{-1}$, where $a \in G$, and $b \in G_{v^{*}}$. By Lemma 2.7, there exists a reduced word $w$ of $G$ such that $o(w)=o\left(w_{1}\right), t(w)=v^{*}$, and $a=[w]$. Then the words $w_{1}$ and $w \cdot b \cdot w^{-1}$ are of the same type and of the same value. Now applying edge reductions on $w \cdot b \cdot w^{-1}$ yields a reduced word of $G$ of length zero which contradicts Lemma 2.7 , or yields a reduced word $w^{\prime}$ of $G$ such that $w^{\prime}$ is not cyclically reduced. This contradicts the first part of the proposition. Hence [ $\left.w_{1}\right]$ is not in any vertex stabilizer of $G$. This completes the proof.

DEFinITION 2.14. An element $g$ of $G$ is called cyclically reduced if $g$ is the value of a cyclically reduced word of $G$. In view of Proposition 2.13, this concept is well defined.

In the next section, we show that some elements of $G$ are conjugate to cyclically reduced elements of $G$ and some not.

3. On invertor elements of groups acting on trees. Throughout this section, $G$ will be a group acting on a tree $X, T$ a tree of representatives for the action of $G$ on $X$ and $Y$ a fundamental domain for the action of $G$ on $X$ such that $X^{G}=\varphi$ and $T \subseteq Y$. We have the following definition.

DeFinition 3.1. Let $g$ be an element of $G$ and $x$ be an edge of $X$. If $g(x)=\bar{x}$, then $g$ is called an invertor element of $G$ and $x$ is called inverted edge under $g$. It is clear that if $x$ is an inverted edge under $g$, then $\bar{x}$ is an inverted edge under $g$ and $g^{2} \in G_{x}$. The main result of this section is the following theorem.

THEOREM 3.2. Let $g$ be an element of $G$. Then the following are equivalent:

(i) $g$ is an invertor element of $G$;

(ii) $g$ is conjugate to an element of the form $[y] a$, where $y$ is an edge of $Y$ such that $G(y, \bar{y}) \neq \varphi$, and $a \in G_{y}$;

(iii) $g^{2}$ is in an edge stabilizer of $G$, and $g$ is not in any vertex stabilizer of $G$;

(iv) $g$ is not conjugate to any cyclically reduced element of $G$;

(v) $|g|_{v}$ is odd and $\left|g^{2}\right|_{v}<|g|_{v}$ for any vertex $v$ of $T$. 
Proof. (i) $\Longrightarrow$ (ii). There exists an edge $x$ of $X$ such that $g(x)=\bar{x}$. The structure of $Y$ implies that there exist an edge $y$ of $Y$ and an element $f$ of $G$ such that $x=f(y)$ and $G(y, \bar{y}) \neq \varphi$. Then $g(x)=\bar{x}$ implies that $g f(y)=f[y](y)$. Hence $g=f[y] a f^{-1}$, where $a \in G_{y}$.

(ii) $\Longrightarrow$ (iii). If $g=f[y] a f^{-1}$, where $y$ is an edge of $Y$ such that $G(y, \bar{y}) \neq \varphi$ and $a \in G_{y}$, then $g^{2} \in G_{x}$, where $x$ is the edge $f(y)$. Now we show that $g$ is not in any vertex stabilizer of $G$. For, if $g$ is in a vertex stabilizer of $G$, then $[y] a \in G_{v}$, where $v$ is a vertex of $X$. Then $[y] a=b c b^{-1}$, where $b \in G$ and $c \in G_{v^{*}}$. Let $w$ be the word $w=1 \cdot y \cdot a$. It is clear that $w$ is a cyclically reduced word of $G$ of type $(o(y))^{*}$, value $[y] a$, and of length one. By Lemma 2.7 , there exists a reduced word $w^{\prime}$ such that $o\left(w^{\prime}\right)=(o(y))^{*}, t\left(w^{\prime}\right)=v^{*}$, and $\left[w^{\prime}\right]=b$.

Let $w_{0}$ be the word $w_{0}=w^{\prime} \cdot c \cdot w^{\prime-1}$. Now applying edge reductions on $w_{0}$ yields a reduced word of $G$ of type $(o(y))^{*}$, value $[w]$, and of even length. Since $w$ is reduced of length 1 , this contradicts Lemma 2.7. Hence $g$ is not in any vertex stabilizer of $G$.

(iii) $\Longrightarrow$ (iv). Let $w$ be a cyclically reduced word of $G$ of type $u, f \in G$, and $v=$ $f(u)$ such that $g=f[w] f^{-1}$. If $|w|=0$, then $[w] \in G_{u}$ and $g \in G_{v}$. This contradicts the assumption that $g$ is not in any vertex stabilizer of $G$. If $|w| \geq 1$, then $w^{2}$ is a cyclically reduced word of $G$ of type $u$, and $\left|w^{2}\right| \geq 2$. This implies that $g^{2}=f\left[w^{2}\right] f^{-1}$. Then $\left[w^{2}\right]=f^{-1} g^{2} f$. Since $g^{2} \in G_{v}$, therefore $\left[w^{2}\right] \in G_{u}$. This contradicts Proposition 2.13.

(iv) $\Longrightarrow$ (v). Let $w$ be a closed and reduced word of $G$ of type $v$ and value $g$. Since $g$ is not conjugate to any cyclically reduced elements of $G$, therefore $w$ can be written as $w=w_{0} \cdot a \cdot y \cdot b \cdot w_{0}^{-1}$, where $w_{0}$ is a reduced word of $G$ such that $o\left(w_{0}\right)=v$, $t\left(w_{0}\right)=(o(y))^{*}$, and $y$ is an edge of $Y$ such that $G(y, \bar{y}) \neq \varphi$ and $a, b \in G_{y}$. Then $g^{2}$ is the value of the word $w_{0} \cdot c \cdot w_{0}^{-1}$, where $c=a[y] b a[y] b$. Now applying edge reductions on $w$ yields a reduced word of $G$ of odd length. Similarly, by applying edge reductions on $w_{0} \cdot c \cdot w_{0}^{-1}$ yields a reduced word of $G$ of even length.

This implies that $|g|_{v}$ is odd and $\left|g^{2}\right|_{v}<|g|_{v}$.

(v) $\Longrightarrow(\mathrm{i}) . g$ is the value of the closed and reduced word $w=w_{0} \cdot y \cdot w_{1}$ of $G$ of type $a$ vertex $v$ of $T$, where $w_{0}$ and $w_{1}$ are reduced words of $G$ such that $\left|w_{0}\right|=\left|w_{1}\right|$, and $y$ is an edge of $Y$. Then $g^{2}$ is the value of $w^{2}$. Since $\left|g^{2}\right|_{v}<|g|_{v}$, therefore $w^{2}$ is not reduced. This implies that $\left[w_{1}\right]=\left[w_{0}\right]^{-1}$, and $G(y, \bar{y}) \neq \varphi$. So $g=\left[w_{0}\right][y]\left[w_{0}\right]^{-1}$. Let $x$ be the edge $x=\left[w_{0}\right](y)$. Then

$$
\begin{aligned}
g(x) & =\left[w_{0}\right][y]\left[w_{0}\right]^{-1}\left(\left[w_{0}\right](y)\right)=\left[w_{0}\right]([y](y)) \\
& =\left[w_{0}\right](\bar{y}), \quad \text { because } G(y, \bar{y}) \neq \varphi \\
& =\overline{\left[w_{0}\right](y)}=\bar{x} .
\end{aligned}
$$

This completes the proof.

REMARK 3.3. We note that the condition $X^{G}=\varphi$ is essential. For instance, if $X^{G} \neq$ $\varphi$, then there exists a vertex $v$ of $X$ such that $G=G_{v}$. Then every invertor element of $G$ is in $G_{v}$. This contradicts Theorem 3.2(iii).

COROLLARY 3.4. Every noninvertor element of $G$ is conjugate to a cyclically reduced element of $G$. 
COROLLARY 3.5. Let $g$ be an element of $G, v$ be a vertex of $\Gamma$, and $n$ be an integer. Then

(i) $\left|g^{2 n}\right|_{v}=\left|g^{2 n+1}\right|_{v}$ if $g \in G_{v}$,

(ii) $\left|g^{2 n}\right|_{v}<\left|g^{2 n+1}\right|_{v}$ if $g$ is an invertor element of $G$,

(iii) $\left|g^{2 n}\right|_{v}>\left|g^{2 n+1}\right|_{v}$ if $g$ is noninvertor element of $G, g \notin G_{v}$.

4. Finitely generated subgroups of groups acting on trees. The main result of this section is the following theorem.

THEOREM 4.1. Let $G$ be a group acting on a tree $X, T$ be a tree of representatives for the action of $G$ on $X$ and $Y$ be a fundamental domain for the action of $G$ on $X$ such that $X^{G}=\varphi$ and $T \subseteq Y$. Let $H$ be a finitely generated subgroup of $G$. Then $H$ contains an invertor element or a conjugate of $H$ contains a cyclically reduced element of length at least one on which $H$ is not in any vertex stabilizer of $G$, or $H$ is in a vertex stabilizer of $G$. If $H$ is contained in the vertex stabilizers $G_{u}$ and $G_{v}$ of the vertices $u$ and $v$ of $X$, then $G_{u}=G_{v}$, or $H$ lies in any edge stabilizer $G_{x}$, where $x$ is any edge in the reduced path in $X$ joining $u$ and $v$.

Proof. If $H$ contains an invertor element of $G$, then by Theorem 3.2(iii), $H$ is not in any vertex stabilizer of $G$. Now assume that $H$ contains no invertor element of $G$. Since $H$ is finitely generated, therefore $H=\left\langle h_{1}, \ldots, h_{n}\right\rangle$. Let $w_{1}, \ldots, w_{n}$ be closed and reduced words of $G$ of the same type such that $\left[w_{i}\right]=h_{i}$ for $i=1, \ldots, n$. The proof is by induction on the sum $r$ of the lengths of the words $w_{1}, \ldots, w_{n}$. If $r=0$, then it is clear that $H$ is contained in $G_{v}$, where $v$ is the type of the words $w_{1}, \ldots, w_{n}$. Now assume that finitely generated subgroups of $G$ having generators in which the sum of the lengths of closed and reduced words of $G$ of the same type and values the generators of these subgroups is less than $r$ are contained in the vertex stabilizers of some vertices of $X$, or have cyclically reduced elements of length at least one. If one of the words $w_{1}, \ldots, w_{n}$ is cyclically reduced of length at least one we are done. Assume that this is not the case.

Let $h \in\left\{h_{1}, \ldots, h_{n}\right\}$ and $w \in\left\{w_{1}, \ldots, w_{n}\right\}$ such that $[w]=h$ and

$$
w=g_{0} \cdot y_{1} \cdot g_{1} \cdots y_{m} \cdot g_{m}, \quad m \geq 1,
$$

$h$ is not cyclically reduced; for, if $h$ is cyclically reduced, then $h \in H$ is cyclically reduced of length at least one. This contradicts the assumption that $H$ has no cyclically reduced element of length at least one. If $h^{\prime} \in\left\{h_{1}, \ldots, h_{n}\right\}$ and $w^{\prime} \in\left\{w_{1}, \ldots, w_{n}\right\}$ such that $\left[w^{\prime}\right]=h^{\prime}$ and $w^{\prime}=f_{0} \cdot x_{1} \cdot f_{1} \cdots x_{s} \cdot f_{s}$, then $h h^{\prime}$ is not cyclically reduced. Otherwise $h h^{\prime}$ will be cyclically reduced of length at least one. This implies that $\left[y_{m}\right] g_{m}=a\left(g_{0}\left[y_{1}\right]\right)^{-1}$, where $a \in G_{+y_{m}}$, and

$$
\left[x_{s}\right] f_{s}=\left(f_{0}\left[x_{1}\right]\right)^{-1}=g_{0}\left[y_{1}\right] a^{-1} c, \quad \text { where } c=\left[y_{m}\right] g_{m} f_{0}\left[x_{1}\right] .
$$

Let $h_{i}^{\prime}=\left(g_{0}\left[y_{1}\right]\right)^{-1} h_{i} g_{0}\left[y_{1}\right]$ for $i=1, \ldots, n$. Then

$$
H=\left(g_{0}\left[y_{1}\right]\right)^{-1} H g_{0}\left[y_{1}\right]=\left\langle h_{1}^{\prime}, \ldots, h_{n}^{\prime}\right\rangle .
$$

Then the elements $h_{1}^{\prime}, \ldots, h_{n}^{\prime}$ are values of closed and reduced words $w_{1}^{\prime}, \ldots, w_{n}^{\prime}$ of $G$ of type $\left(t\left(y_{1}\right)\right)^{*}$ and $\left[w_{i}\right]=h_{i}^{\prime}$ for $i=1, \ldots, n$. Then the sum of the lengths of $w_{1}^{\prime}, \ldots, w_{n}^{\prime}$ 
is less than the sum of the lengths of the words $w_{1}, \ldots, w_{n}$. Then by hypothesis assumption on $r$, either $H^{\prime}$ is contained in $G_{z}$ for some $z \in V(X)$, or $H^{\prime}$ contains a cyclically reduced element of length at least one. If $H^{\prime}$ is contained in $G_{z}$, then it is clear that $H$ is contained in $G_{v}$, where $v=g_{0}\left[y_{1}\right](z)$. Otherwise $H^{\prime}$ is a conjugate of $H$ and $H^{\prime}$ contains a cyclically reduced element of length at least one. Now assume that $H$ is contained in the vertex stabilizers $G_{u}$ and $G_{v}$ of the vertices $u$ and $v$ of $X$ such that $G_{u} \neq G_{v}$. Then it is clear that $u \neq v$, and there exists a unique reduced path $x_{1}, \ldots, x_{n}$ in $X$ joining $u$ and $v$. Consequently $G_{u} \cap G_{v} \subseteq \bigcap_{i=1}^{n} G_{x_{i}}$, and $H$ lies in any edge stabilizer $G_{x}$, where $x \in\left\{x_{1}, \ldots, x_{n}\right\}$.

This completes the proof.

COROLLARY 4.2. If $H$ is a finite subgroup of $G$, then $H$ contains an invertor element, or $H$ is in a vertex stabilizer of $G$.

Proof. If $H$ contains an invertor element, then $H$ is not in any vertex stabilizer of $G$. If a conjugate of $H$ contains a cyclically reduced element $g$ of length at least one, then $g^{n}$ is a cyclically reduced element of length at least one for any integer $n$. Then by Proposition $2.13, g$ is not in any vertex stabilizer of $G$. Thus $g$ is of infinite order. Hence $H$ is in a vertex stabilizer of $G$. This completes the proof.

COROLLARY 4.3. If $H$ is a cyclic subgroup of $G$ generated by the element $g$ such that $g$ is not in any vertex stabilizer of $G$, and $H \cap G_{x}$ is trivial for any edge of $X$, then $H$ is cyclic of order 2 if $g$ is an invertor element, or $H$ is infinite cyclic if $g$ is not invertor element.

Proof. If $g$ is an invertor element, then by Theorem 3.2(iii), $g^{2}$ is in an edge stabilizer of $G$. By assumption $g^{2}=1$, the identity element of $G$. Hence $H$ is a cyclic of order 2. If $g$ is not invertor element, then from above $g$ is of infinite order. Hence $H$ is infinite cyclic. This completes the proof.

5. Applications. This section is an application of Theorems 3.2 and 4.1. Groups acting on trees can be divided into two parts. The first part is of action fixing some vertices, and the other not. For groups acting on trees without fixing any vertices are some of actions without inversions, and the other with inversions. Free groups, free product of groups, free product of groups with amalgamation subgroup, tree product of groups, and HNN groups are examples of groups acting on trees without inversions. A new class of groups called quasi $H N N$ groups are examples of groups acting on trees with inversions. In fact, free product of groups, free product of groups with amalgamation subgroup are special cases of tree product of groups, and, free groups and $H N N$ groups are special cases of quasi $H N N$ groups as we will see below. We start with tree product of groups. For more details of tree product of groups we refer the readers to Fisher [1] or Karrass and Solitar [2].

Proposition 5.1. Let $G=\prod_{i \in I}^{*}\left(A_{i}, U_{j k}=U_{k j}\right)$ be a nontrivial tree product of the groups $A_{i}, i \in I$, and $H$ be a finitely generated subgroup of $G$. Then a conjugate of $H$ contains a cyclically reduced element of length at least one or $H$ is contained in a conjugate of $A_{i}, i \in I$. Moreover, if $H$ is finite, then $H$ is contained in a conjugate of $A_{i}, i \in I$. 
Proof. By Mahmood [8], there exists a tree $X$ on which $G$ acts without inversions such that any tree of representatives for the action of $G$ on $X$ equals the corresponding fundamental domain for the action of $G$ on $X, X^{G}=\varphi$, and for every vertex $v$ of $X$, and every edge $x$ of $X, G_{u}$ is isomorphic to $A_{i}, i \in I$, and $G_{X}$ is isomorphic to $U_{i k}$, for some $i, k \in I$. Moreover, $G$ contains no invertor elements. Therefore by Theorem 4.1, the proof of Proposition 5.1 follows.

COROLLARY 5.2. If $G$ is a free product with amalgamation group, then for any finitely generated subgroup $H$ of $G$ a conjugate of $H$ contains a cyclically reduced element of length at least one or $H$ is contained in a conjugate of a factor of $G$. Moreover if $H$ is finite, then $H$ is contained in a conjugate of factor of $G$.

Before we state our next proposition we introduce the concept of quasi $H N N$ groups. Khanfar and Mahmood [3] extended the class of $H N N$ groups to a new class of groups called quasi $H N N$ groups defined as follows.

Let $G$ be a group, $\left\{A_{i}: i \in I\right\},\left\{B_{i}: i \in I\right\}$, and $\left\{C_{j}: j \in J\right\}$ be families of subgroups of $G$. For each $i \in I$, let $\phi_{i}: A_{i} \rightarrow B_{i}$ be an onto isomorphism, and for each $j \in J$ let $\alpha_{j}: C_{j} \rightarrow C_{j}$ be an outer automorphism of order 2 such that the inner automorphism $\alpha_{j}^{2}$ is determined by $\bar{c}_{j} \in C_{j}$ fixed by $\alpha_{j}$. That is, $\alpha_{j} \in \operatorname{Out}\left(C_{j}\right)$ and $\alpha_{j}^{2} \in \operatorname{Inn}\left(C_{j}\right)$ such that $\alpha_{j}\left(\bar{c}_{j}\right)=\bar{c}_{j}$ and $\alpha_{j}^{2}(c)=\bar{c}_{j} c \bar{c}_{j}^{-1}$ for all $c \in C_{j}$.

Let $G^{*}$ be the group of the presentation

$$
G^{*}=\left(G, t_{i}, t_{j} \mid \operatorname{rel} G, t_{i} A_{i} t_{i}^{-1}=B_{i}, t_{j} C_{j} t_{j}^{-1}=C_{j}, t_{j}^{2}=\bar{c}_{j}, i \in I, j \in J\right)
$$

where $t_{i} A_{i} t_{i}^{-1}=B_{i}$ stands for the set of relations $t_{i} a_{i} t_{i}^{-1}=\phi_{i}\left(a_{i}\right)$ for all $a_{i} \in A_{i}$, and $t_{j} C_{j} t_{j}^{-1}=C_{j}$ stands for the set of relations $t_{j} c_{j} t_{j}^{-1}=\alpha_{j}\left(c_{j}\right)$ for all $c_{j} \in C_{j}$. The group $G^{*}$ is called a quasi $H N N$ group of base $G$ and associated pairs $\left(A_{i}, B_{i}\right)$, and $\left(C_{j}, C_{j}\right)$, $i \in I, j \in J$ of subgroups of $G$. Moreover, $G$ is embedded in $G^{*}$. We note that if $J=\varnothing$, then $G^{*}$ is an $H N N$ group of base $G$ and associated pairs $\left(A_{i}, B_{i}\right), i \in I$ of subgroups of $G$.

Proposition 5.3. Let $G^{*}$ be the quasi $H N N$ group of base $G$ and associated pairs $\left(A_{i}, B_{i}\right)$, and $\left(C_{j}, C_{j}\right), i \in I, j \in J$ of subgroups of $G$ defined above. Let $H$ be a finitely generated subgroup of $G^{*}$. Then $H$ contains an element conjugate to an element $t_{j}$, $j \in J$ or $H$ contains a cyclically reduced element of length at least one or $H$ is contained in a conjugate of $G$. Moreover, if $H$ is finite containing no element conjugate to the element $t_{j}, j \in J$, then $H$ is contained in a conjugate of $G$.

Proof. By Mahmood and Khanfar [9], there exists a tree $X$ on which $G^{*}$ acts with inversions such that $G^{*}$ is transitive on the set $V(X), X^{G^{*}}=\varphi$, and for every vertex $v$ of $X$, and every edge $x$ of $X, G_{v}^{*}$ is isomorphic to $G$, and $G_{x}^{*}$ is isomorphic to $A_{i}, i \in I$, or is isomorphic to $C_{j}, j \in J$. Moreover, $G^{*}$ contains the invertor elements conjugate to an element $t_{j}, j \in J$. Therefore by Theorem 4.1, the proof of Proposition 5.3 follows.

COROLLARY 5.4. For any finitely generated subgroup $H$ of an HNN group $G^{*}$ of base $G$, a conjugate of $H$ contains a cyclically reduced element of length at least one 
or $H$ is contained in a conjugate of $G$. Moreover if $H$ is finite, then $H$ is contained in $a$ conjugate of $G$.

Proof. By taking $J=\varnothing$, we see that $G^{*}$ of Proposition 5.3 above is an $H N N$ group containing no invertor elements. Therefore the proof of Corollary 5.4 follows from Proposition 5.3.

\section{REFERENCES}

[1] J. Fisher, The subgroups of a tree product of groups, Trans. Amer. Math. Soc. 210 (1975), 27-50. Zbl 323.20027.

[2] A. Karrass and D. Solitar, The subgroups of a free product of two groups with an amalgamated subgroup, Trans. Amer. Math. Soc. 150 (1970), 227-255. MR 41\#5499. Zbl 223.20031.

[3] M. I. Khanfar and R. M. S. Mahmood, On quasi HNN groups, submitted to Kuwait J. Sci. Engrg.

[4] _ A note on groups acting on connected graphs, J. Univ. Kuwait Sci. 16 (1989), no. 2, 205-208. MR 91a:05051. Zbl 691.05018.

[5] R. C. Lyndon and P. E. Schupp, Combinatorial group theory, Springer-Verlag, Berlin-New York, 1977. MR 58\#28182. Zbl 368.20023.

[6] R. M. S. Mahmood, Presentation of groups acting on trees with inversions, Proc. Roy. Soc. Edinburgh Sect. A 113 (1989), no. 3-4, 235-241. MR 91b:20037. Zbl 695.20019.

[7] _ The normal form theorem of groups acting on trees with inversions, J. Univ. Kuwait Sci. 18 (1991), 7-16. MR 93c:20050. Zbl 751.20019.

[8] _ - The subgroup theorem for groups acting on trees, Kuwait J. Sci. Engrg. 25 (1998), no. 1, 17-33. MR 99g:20046. Zbl 905.20015.

[9] R. M. S. Mahmood and M. I. Khanfar, On subgroups of quasi HNN groups, submitted to Internat. J. Math. Math. Sci.

[10] J. P. Serre, Arbres, Amalgames, SL2, Société Mathématique de France, Paris, 1977, Avec un sommaire anglais. Redige avec la collaboration de Hyman Bass.; Asterisque, No. 46. MR 57\#16426. Zbl 369.20013.

Mahmood: Ajman University of Science and Technology, ABu Dhabi, United ARAB EMIRATES

E-mail address: rasheed75@hotmai 1 .com

KHANFAR: DePARTMENT OF MATHEMATICS, YARMOUK UNIVERSITY, IRBID, JORDAN 


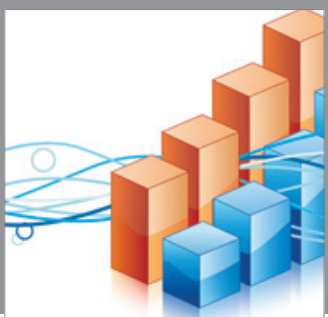

Advances in

Operations Research

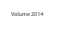

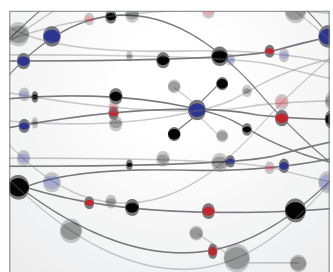

\section{The Scientific} World Journal
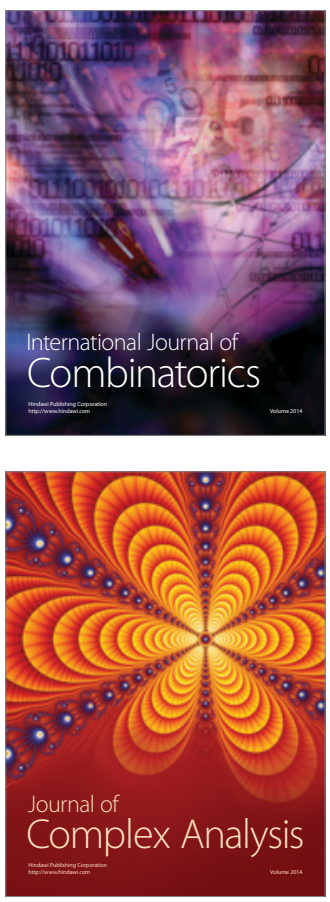

International Journal of

Mathematics and

Mathematical

Sciences
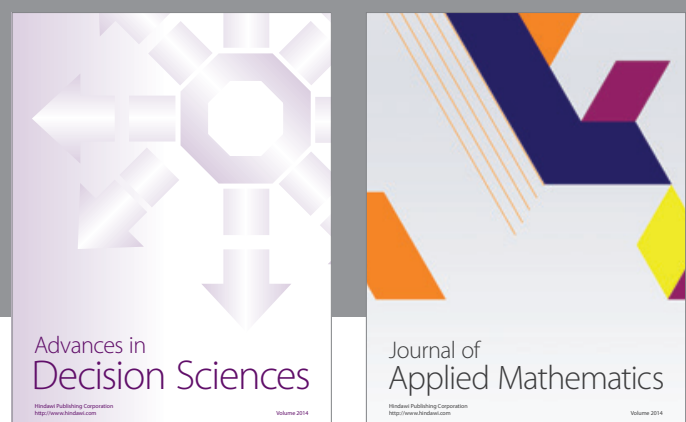

Journal of

Applied Mathematics
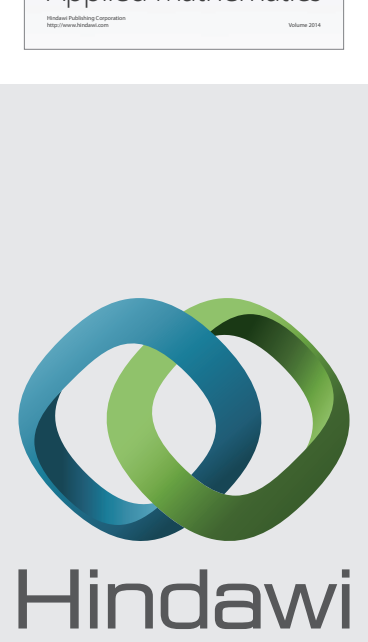

Submit your manuscripts at http://www.hindawi.com
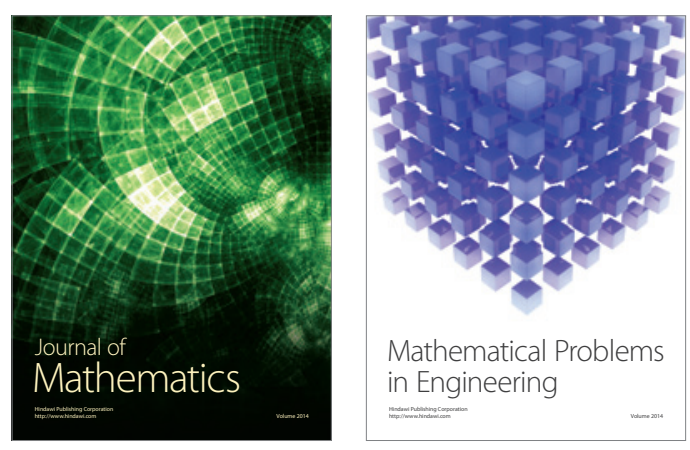

Mathematical Problems in Engineering
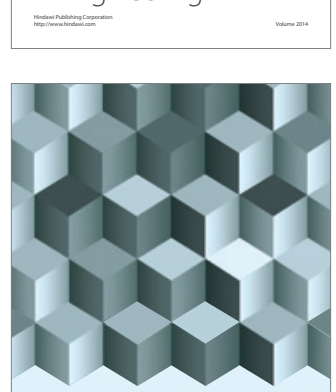

Journal of

Function Spaces
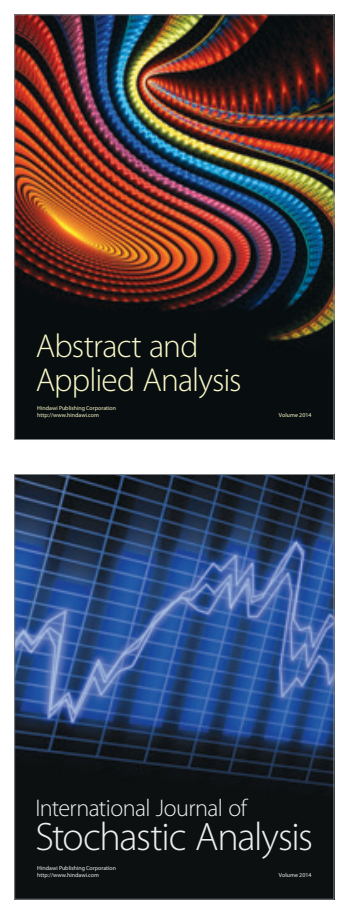

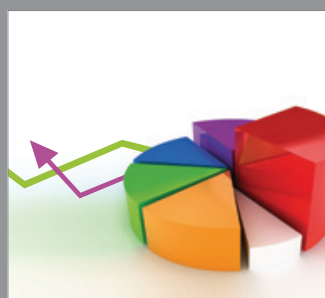

ournal of

Probability and Statistics

Promensencen
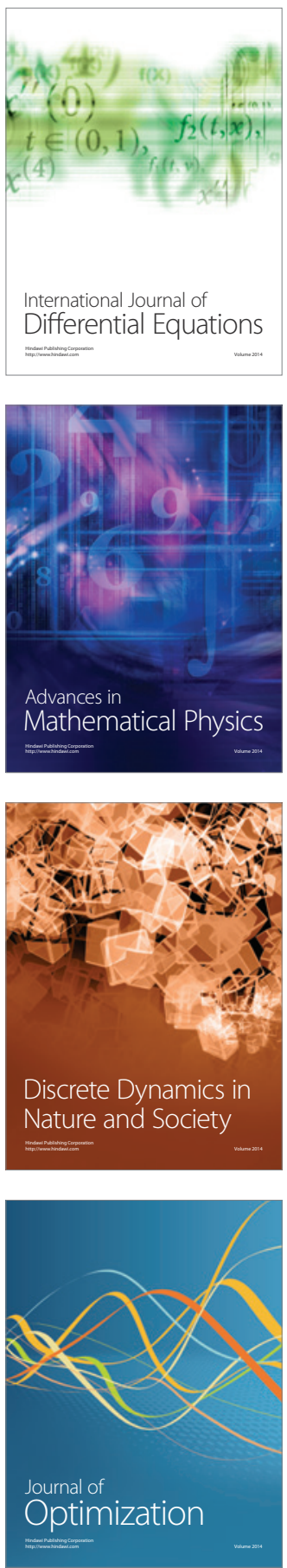